\title{
Five-loop Konishi from the mirror TBA
}

\section{Gleb Arutyunov, ${ }^{a}$ Sergey Frolov $^{b, 1}$ and Ryo Suzuki ${ }^{b}$}

${ }^{a}$ Institute for Theoretical Physics and Spinoza Institute, Utrecht University, 3508 TD Utrecht, The Netherlands

${ }^{b}$ Hamilton Mathematics Institute and School of Mathematics, Trinity College, Dublin 2, Ireland

E-mail: G.E.Arutyunov@uu.nl, frolovs@maths.tcd.ie, rsuzuki@maths.tcd.ie

Abstract: We use the Thermodynamic Bethe Ansatz equations for the $\mathrm{AdS}_{5} \times \mathrm{S}^{5}$ mirror model to derive the five-loop anomalous dimension of the Konishi operator. We show numerically that the corresponding result perfectly agrees with the one recently obtained via the generalized Lüscher formulae. This constitutes an important test of the AdS/CFT TBA system.

KeYwords: AdS-CFT Correspondence, Integrable Field Theories

ARXIV EPRINT: 1002.1711

\footnotetext{
${ }^{1}$ Correspondent fellow at Steklov Mathematical Institute, Moscow.
} 


\section{Contents}

1 Introduction 1

2 Linearizing the TBA system 2

3 Correction to the asymptotic Bethe equations 6

\section{Introduction}

The mirror Thermodynamic Bethe Ansatz (TBA) is generalization of the relativistic TBA [1] and offers a tool to determine the spectrum of the $\operatorname{AdS}_{5} \times \mathrm{S}^{5}$ superstring [2, 3]. Recently there has been interesting progress on its precise formulation and in deriving some consequences of the corresponding spectral equations [4]-[14]. In parallel development the four-loop anomalous dimension of the Konishi operator was obtained [15] by means of the generalized Lüscher formulae $[15-17]^{1}$ and it exhibits a perfect match with a direct field-theoretic computation [28, 29]. More recently, a refined version of the generalized Lüscher formula has been proposed, and applied to the five-loop anomalous dimension of the Konishi operator [30],

$$
\Delta^{(10)}=\Delta_{\text {asympt }}^{(10)}+g^{10}\left\{-\frac{81 \zeta(3)^{2}}{16}+\frac{81 \zeta(3)}{32}-\frac{45 \zeta(5)}{4}+\frac{945 \zeta(7)}{32}-\frac{2835}{256}\right\},
$$

where $g$ is the coupling constant (string tension) related to the 't Hooft coupling $\lambda$ through $\lambda=4 \pi^{2} g^{2}$. This result has been further generalized to all twist two operators by also invoking the reciprocity principle [31]. Although no field-theoretical computation has been done at five loops, those anomalous dimension being continued to the negative values of spin enjoy a quite non-trivial agreement with the constraints imposed by the BFKL equation [32]-[35].

Having in mind all these developments, it is time to ask whether the proposed TBA spectral equations are in accord with the perturbative findings based on the generalization of Lüscher's approach. As for Konishi at four loops, incorporation of the corresponding result in the TBA framework does not pose any real difficulty because the leading finite-size correction has been built-in when the excited-state TBA is formulated; all the contribution to the anomalous dimension (the energy of the corresponding string state) comes from the main $Y_{Q}$-functions taken at their asymptotic values [9], the latter being given by the generalized Lüscher formulae constructed through the infinite-volume scattering data [30]. Also, rapidities $u_{k}, k=1, \ldots, N$, of the excited string particles forming an $N$-particle state under consideration are determined by the asymptotic Bethe Ansatz (ABA) equations (also known as the Bethe-Yang equations).

\footnotetext{
${ }^{1}$ See [18]-[30] for other interesting applications of Lüscher's approach.
} 
The situations change, however, for the five-loop case. As was argued in [15, 30], to find the anomalous dimension of Konishi at five loops, one has to compute the correction to the ABA, though still the asymptotic $Y_{Q}$-functions can be used. The exact Bethe equation determines the shift $\delta u_{k}$ of particle rapidities $u_{k}=u_{k}^{o}+g^{8} \delta u_{k}$ from their asymptotic values $u_{k}^{o}$ as

$$
\sum_{j=1}^{M} \frac{\delta \mathrm{ABA}\left(u_{k}\right)}{\delta u_{j}} \delta u_{j}+\Phi_{k}^{(8)}=0 .
$$

The first term here is a variation of the ABA equations and the term $\Phi^{(8)}$ is the leading finite-size correction to the ABA of order $g^{8}$. The formula above should be evaluated at $u_{k}=u_{k}^{(2)}$, where $u_{k}^{(2)}$ are the one-loop values of the particle rapidities. As soon as $\delta u_{k}$ are determined, the five-loop correction to the dimension (energy of the corresponding string state) follows from expanding the exact dispersion relation up to the five-loop order, i.e. up to $g^{10}$.

It turns out that the correction $\Phi^{(8)}$ derived through analytic continuation of the mirror TBA equation looks rather different from the one derived through Lüscher-type perturbative arguments [30]. The difference occurs due to the fact that the exact Bethe equations involve auxiliary Y-functions which must satisfy a coupled system of the TBA equations. Starting from five loops, the auxiliary Y-functions start to contribute nontrivially to the modification of the ABA.

In this note we find a strong evidence that the results obtained for the Konishi operator from both the TBA and Lüscher approaches are in an excellent agreement at the fiveloop level. Fortunately, as discussed above, what we have to do is to show only that the correction to the ABA derived from the TBA agrees with $\Phi^{(8)}$ found from Lüscher's approach [30]. This will be done by expanding the TBA equations around the asymptotic solution [9] and by linearizing the exact Bethe equations around the ABA. As we will see, the exact Bethe equations at order $g^{8}$ involve a leading correction to a single auxiliary function $Y_{1 \mid v w}$, which we will determine from the linearized TBA equations numerically. Then, using this result for $Y_{1 \mid v w}$ we evaluate numerically the correction to the exact Bethe equations and find that it agrees with $\Phi^{(8)}$ of [30] with a sufficiently high precision. It would be important to support this numerical agreement by an analytic proof.

The paper is organized as follows. In the next section we present the linearization of the simplified TBA equations. We use them in section 3 to compute numerically the correction to the exact Bethe equations finding an agreement with the corresponding result in [30].

\section{Linearizing the TBA system}

In the light-cone gauge the string vacuum corresponds to the gauge theory operator $\operatorname{tr} Z^{J}$, where $Z$ is one of the three complex scalars of the $\mathcal{N}=4$ super Yang-Mills theory. In this work we will be interested in the excited states from the $\mathfrak{s l}(2)$-sector, the latter comprises the composite operators of the type $\operatorname{tr} D^{N} Z^{J}$, where $D$ is a light-cone derivative. For $J=2$ these are operators of twist two spin $N$. In particular, the operator with $J=2$ and $N=2$ is the $\mathfrak{s l}(2)$ descendent of the Konishi operator. 
The Thermodynamic Bethe Ansatz approach for the $\mathrm{AdS}_{5} \times \mathrm{S}^{5}$ mirror model leads to the following expression for the energy of the corresponding $N$-particle string states

$$
E=J+\sum_{i=1}^{N} \mathcal{E}\left(p_{i}\right)-\frac{1}{2 \pi} \sum_{Q=1}^{\infty} \int_{-\infty}^{\infty} \mathrm{d} u \frac{d \widetilde{p}^{Q}}{d u} \log \left(1+Y_{Q}\right) .
$$

Here $J$ is the angular momentum carried by the string rotating around the equator of $\mathrm{S}^{5}$. The integration runs over a real rapidity line of the mirror theory, $\widetilde{p}^{Q}$ and $Y_{Q}$ are momenta and Y-functions of the mirror $Q$-particles. The asymptotic energies of string theory particles with momenta $p_{i}$ are fixed by the dispersion relation $[36,37]$

$$
\mathcal{E}(p)=\sqrt{1+4 g^{2} \sin ^{2} \frac{p}{2}}
$$

The function $Y_{Q}$ is exponentially small at large $J$. Therefore the last term of (2.1) can be regarded as a finite-size correction to the asymptotic spectrum of string energies.

For a fixed $J$ and small $g$, the $Y_{Q}$ functions become small again. The finite-size corrections provide so-called wrapping corrections to the energy or the anomalous dimension at weak coupling. In particular, for the Konishi operator the finite-size effects make their appearance starting from $g^{8}$ that corresponds to the fourth loop order of perturbation theory [15].

Recall that the large $J$ asymptotic solution of the excited-state TBA equations can correctly reproduce the leading finite-size corrections to the energy. The TBA equations are formulated in terms of the following Y-functions: $Y_{Q}$-functions associated with $Q$-particle bound states, auxiliary functions $Y_{Q \mid v w}$ for $Q \mid v w$-strings, $Y_{Q \mid w}$ for $Q \mid w$-strings, and $Y_{ \pm}$for $y_{ \pm}$-particles $[4,5]$.

In what follows it is convenient to use the simplified TBA equations derived in $[5,6,14]$. To determine the leading finite-size correction to the asymptotic form of $Y$-functions, we linearize the simplified TBA equations by introducing for any Y-function the following representation

$$
Y=Y^{o}(1+\mathscr{Y}),
$$

where $Y^{o}$ is the corresponding asymptotic expression and $\mathscr{Y}$ is treated as the perturbation. The linearized TBA equations will then take the following form

- $M \mid w$-strings: $M \geq 1, \mathscr{Y}_{0 \mid w}=0$

$$
\mathscr{Y}_{M \mid w}=\left(A_{M-1 \mid w} \mathscr{Y}_{M-1 \mid w}+A_{M+1 \mid w} \mathscr{Y}_{M+1 \mid w}\right) \star s+\delta_{M 1}\left(\frac{\mathscr{Y}_{+}}{1-Y_{+}^{o}}-\frac{\mathscr{Y}_{-}}{1-Y_{-}^{o}}\right) \hat{\star} s,
$$

where we have introduced the concise notation $A_{M \mid w}=\frac{Y_{M \mid w}^{o}}{1+Y_{M \mid w}^{o}}$.

- $M \mid v w$-strings: $M \geq 1, \mathscr{Y}_{0 \mid v w}=0$

$$
\begin{aligned}
\mathscr{Y}_{M \mid v w}=\left(A_{M-1 \mid v w} \mathscr{Y}_{M-1 \mid v w}+A_{M+1 \mid v w} \mathscr{Y}_{M+1 \mid v w}\right) \star s-Y_{M+1}^{o} \star s & \\
& +\delta_{M 1}\left(\frac{\mathscr{Y}_{-}}{1-\frac{1}{Y_{-}^{o}}}-\frac{\mathscr{Y}_{+}}{1-\frac{1}{Y_{+}^{o}}}\right) \hat{\star} s .
\end{aligned}
$$


Here we also defined $A_{M \mid v w} \equiv \frac{Y_{M \mid v w}^{o}}{1+Y_{M \mid v w}^{o}}$. Any asymptotic Y-functions except for $Y_{Q}^{\circ}$ have the magnitude of order 1 , so $A_{M \mid w}, A_{M \mid v w}$ are not small. Note that the Yfunction $Y_{M}^{o}$ provides the leading large $J$ correction to the asymptotic TBA equations and, for this reason, it enters the last equation as an inhomogeneous term.

- $y$-particles

$$
\begin{aligned}
& \mathscr{Y}_{+}-\mathscr{Y}_{-}=Y_{Q}^{o} \star K_{Q y}, \\
& \mathscr{Y}_{+}+\mathscr{Y}_{-}=2\left(A_{1 \mid v w} \mathscr{Y}_{1 \mid v w}-A_{1 \mid w} \mathscr{Y}_{1 \mid w}\right) \star s-Y_{Q}^{o} \star s+2 Y_{Q}^{o} \star K_{x v}^{Q 1} \star s .
\end{aligned}
$$

We will not need the equations for $Q$-particles, because the asymptotic solution for $Y_{Q}$ is already known from the generalized Lüscher's formulas. The equations for $Q$-particles suggest that the next corrections to $Y_{Q}^{o}$ start from $g^{16}$.

The linearized TBA equations above define the leading, exponential in $J$ correction to the asymptotic Y-functions. However, by further fixing $J=2$ we would like to view $\mathscr{Y}$ as another source of perturbative correction in $g$ to the asymptotic Y-functions. Therefore it makes sense to expand further the asymptotic Y-functions in the linearized TBA equations in powers of $g$ having in mind that expansion of $Y_{M}$ starts from $g^{8}$.

The rapidities $u_{k}$ of the Konishi operator are expanded at small $g$ as $u_{1}=-u_{2}=$ $\frac{1}{\sqrt{3} g}\left[1+2 g^{2}-\frac{5 g^{4}}{4}+\mathcal{O}\left(g^{6}\right)\right]$ using the notation of [14]. It is convenient to rescale them as $u_{k} \rightarrow u_{k} / g$ and do the same $u \rightarrow u / g$ with the argument $u$ of the $Y_{M}^{o}(u), Y_{M \mid v w}^{o}(u)$ and $Y_{M \mid w}^{o}(u)$. On the other hand, the functions $Y_{ \pm}^{o}(u)$ are supported on the segment $u \in[-2,2]$ and as for them, the variable $u$ will be kept unrescaled.

First, let us consider the equation for $y$-particles. Performing the change of variables $u \rightarrow u / g$ in the convolution term $Y_{Q}^{o} \star s$ and expanding in $g$, we see that

$$
Y_{Q}^{o} \star s=\int_{-\infty}^{\infty} \frac{\mathrm{d} u}{g} Y_{Q}^{o}\left(\frac{u}{g}\right) \frac{g}{4 \cosh \frac{\pi g}{2}\left(\frac{u}{g}-\frac{v}{g}\right)}=\int_{-\infty}^{\infty} \mathrm{d} u \frac{Y_{Q}^{o}(u)}{4 \cosh \frac{\pi}{2}(u-v)},
$$

where the rescaled Y-function $Y_{Q}^{o}(u)$ reads as

$$
\begin{gathered}
Y_{Q}^{o}(u)=g^{8} \frac{64 Q^{2}\left[-1+Q^{2}+u^{2}-w^{2}\right]^{2}}{\left(Q^{2}+u^{2}\right)^{4}\left[(Q-1)^{2}+(u-w)^{2}\right]\left[(Q+1)^{2}+(u-w)^{2}\right]} \times \\
\times \frac{1}{\left[(Q-1)^{2}+(u+w)^{2}\right]\left[(Q+1)^{2}+(u+w)^{2}\right]}+\mathcal{O}\left(g^{10}\right),
\end{gathered}
$$

and we denote by $w \equiv u_{1}=-u_{2}=\frac{1}{\sqrt{3}}+\mathcal{O}\left(g^{2}\right)$ the rescaled rapidity of the two-particle state corresponding to the Konishi operator. Thus, $Y_{Q} \star s$ starts at order $g^{8}$ in the weakcoupling expansion. Analogously, one can establish that in eq. (2.6) at $g \rightarrow 0$ one has $Y_{Q}^{o} \star K_{Q y} \sim g^{9}$, while in eq. (2.7) one finds $Y_{Q}^{o} \star K_{x v}^{Q 1} \star s \sim g^{8}$. As a consequence, eqs. (2.6) and (2.7) imply that the first non-trivial corrections $\mathscr{Y}_{ \pm}$start at order $g^{8}$, although for the difference one has $\mathscr{Y}_{+}-\mathscr{Y}_{-} \sim g^{9}$.

In fact, there is a general pattern behind inherited from the asymptotic solution for $Y_{ \pm}^{o}$ — the sum $\mathscr{Y}_{+}+\mathscr{Y}_{-}$admits an expansion in even powers of $g$, while the difference $\mathscr{Y}_{+}-\mathscr{Y}_{-}$ 
in odd. Another interesting property is that $\mathscr{Y}_{+}(g)=\mathscr{Y}_{-}(-g)$. Thus, in the perturbative expansion, the coefficients of $\mathscr{Y}_{ \pm}$in front of even powers of $g$ coincide.

Now we turn our attention to eq. (2.5). The notation $\hat{\star}$ signifies that integration in the corresponding convolution term is over the segment $[-2,2]$. Due to the fact that we do not rescale the integration variable in the integrals involving $\mathscr{Y}_{ \pm}$, the expansion of $s$ in this convolution term starts from $g$. Moreover, one finds that at the leading order in $g$ :

$$
Y_{+}^{o}=Y_{-}^{o}=\frac{w^{2}-3}{w^{2}+1}
$$

which is a $u$-independent quantity. Hence,

$$
\left(\frac{\mathscr{Y}_{-}}{1-\frac{1}{Y_{-}^{o}}}-\frac{\mathscr{Y}_{+}}{1-\frac{1}{Y_{+}^{o}}}\right) \hat{\star} s \stackrel{g \rightarrow 0}{=} \frac{1}{4}\left(1+w^{2}\right)\left(\mathscr{Y}_{-}-\mathscr{Y}_{+}\right) \hat{\star} s \sim g^{10},
$$

and, therefore this term produces higher than the leading $g^{8}$ contribution into the equation for $\mathscr{Y}_{M \mid v w}$.

Analogous consideration can be applied to eq. (2.4) which shows that the correction $\mathscr{Y}_{M \mid w}$ starts from the order $g^{10}$. Thus, at order $g^{8}$ we have the following equations to be considered

- $M \mid v w$-strings: $M \geq 1, \mathscr{Y}_{0 \mid v w}=0$

$$
\mathscr{Y}_{M \mid v w}=\left(A_{M-1 \mid v w} \mathscr{Y}_{M-1 \mid v w}+A_{M+1 \mid v w} \mathscr{Y}_{M+1 \mid v w}\right) \star s-Y_{M+1}^{o} \star s .
$$

- $y$-particles $\mathscr{Y}_{+}=\mathscr{Y}_{-}$

$$
2 \mathscr{Y}_{+}=2 A_{1 \mid v w} \mathscr{Y}_{1 \mid v w} \star s-Y_{Q}^{o} \star s+2 Y_{Q}^{o} \star K_{x v}^{Q 1} \star s,
$$

where all the TBA kernels are taken at their leading order. In fact, as we will see below, the correction to the asymptotic Bethe-Yang equations involves at order $g^{8}$ the functions $\mathscr{Y}_{M \mid v w}$ alone, and therefore, the only equation we have to solve is (2.11).

Eq. (2.11) can be equivalently written as

$$
\mathscr{Y}_{Q \mid v w} \star \Omega_{Q M}=-Y_{M+1}^{o} \star s .
$$

Here we defined a kernel

$$
\begin{aligned}
\Omega_{Q M}(u, v)= & \delta_{Q M} \delta(u-v) \\
& -\delta_{Q, M-1} A_{M-1 \mid v w}(u) s(u-v)-\delta_{Q, M+1} A_{M+1 \mid v w}(u) s(u-v),
\end{aligned}
$$

where

$$
\begin{aligned}
A_{M \mid v w}(u)= & \frac{M(M+2)}{(M+1)^{2}} \times \\
& \times\left(1+\frac{1+w^{2}}{\sqrt{M(M+2)-w^{2}}} \frac{1}{u^{2}+\rho_{+}^{2}}-\frac{1+w^{2}}{\sqrt{M(M+2)-w^{2}}} \frac{1}{u^{2}+\rho_{-}^{2}}\right)+\mathcal{O}\left(g^{2}\right),
\end{aligned}
$$




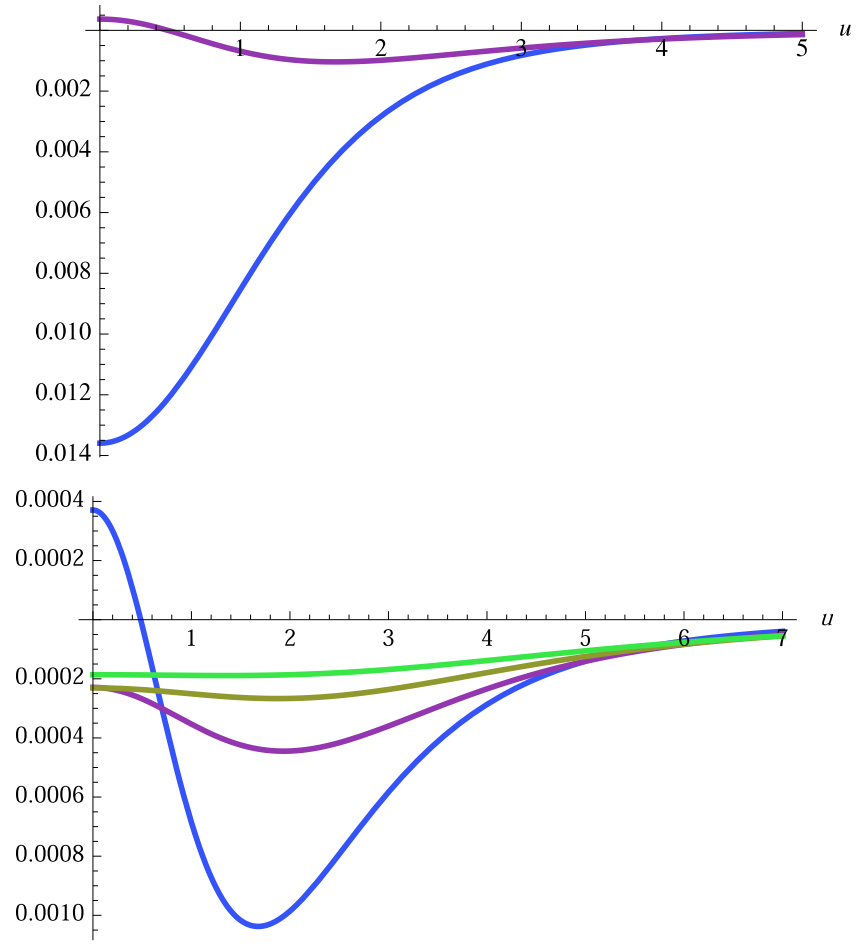

Figure 1. In the upper picture the profiles of the functions $\mathscr{Y}_{1 \mid v w} / g^{8}$ (blue) and $\mathscr{Y}_{2 \mid v w} / g^{8}$ (purple) are depicted. They are obtained by solving numerically eq. (2.13). The lower picture contains the profiles of $\mathscr{Y}_{M \mid v w} / g^{8}$ for $M=2,3,4,5$. The absolute value of $\mathscr{Y}_{M \mid v w}$ decreases as $M$ increases.

with $\rho_{ \pm}^{2}=\left(1 \pm \sqrt{M(M+2)-w^{2}}\right)^{2}$ and $s(u)=\frac{1}{4 \cosh \frac{\pi u}{2}}$. The formal solution to eq. (2.13) can be given with the help of the inverse kernel $\Omega^{-1}$

$$
\mathscr{Y}_{Q \mid v w}=-Y_{M+1}^{o} \star s \star \Omega_{M Q}^{-1} .
$$

The kernel $\Omega$ can be inverted by the power series expansion and computed numerically. This gives rise to a numerical determination of the correction $\mathscr{Y}_{1 \mid v w}$. Alternatively, eq. (2.13) can be solved by iterations, and it is what we have used.

We studied the following inhomogeneous linear equation

$$
\mathscr{Y}_{M \mid v w}^{[n]}=\left(A_{M-1 \mid v w} \mathscr{Y}_{M-1 \mid v w}^{[n]}+A_{M+1 \mid v w} \mathscr{Y}_{M+1 \mid v w}^{[n]}\right) \star s-\delta_{n, M+1} Y_{M+1}^{o} \star s,
$$

which can be solved by numerical iterations starting with $\mathscr{Y}_{M \mid v w}^{[n]}=0$ for each $n=2,3, \ldots$. Since all equations are linear, the solution of the original equation (2.13) is given by the sum $\mathscr{Y}_{M \mid v w} \equiv \sum_{n=2}^{\infty} \mathscr{Y}_{M \mid v w}^{[n]}$. The solution is unambiguously well-defined provided that the homogeneous equation has only the trivial solution $\mathscr{Y}_{M \mid v w}=0$. The profiles of the first few $\mathscr{Y}_{M \mid v w}$ are presented in figure 1.

\section{Correction to the asymptotic Bethe equations}

The exact Bethe equations determine the exact (non-asymptotic) positions of the Bethe roots $u_{k}$. As was shown in [14], for Konishi-like states and below the first critical point the 
exact Bethe equations ${ }^{2}$ admit the following representation

$$
\begin{aligned}
\pi\left(2 n_{k}+1\right)= & L p_{k}+i \sum_{j=1}^{N} \log S_{\mathfrak{s l}(2)}^{1 * 1 *}\left(u_{j}, u_{k}\right)+\operatorname{Im} U \\
& -2 \sum_{M=1}^{\infty} \log \left(1+Y_{M}\right) \star\left(\operatorname{Im} K_{M 1_{*}}^{\Sigma}-s \star \operatorname{Im} K_{v w x}^{M-1,1_{*}}\right) \\
& +2 \log \left(1+Y_{1 \mid v w}\right) \star\left(\operatorname{Im} \tilde{s}-i s \hat{\star} K^{y 1_{*}}\right) \\
& -2 \log \frac{1-Y_{-}}{1-Y_{+}} \hat{\star} s \star \operatorname{Im} K_{v w x}^{11_{*}}-i \log \left(1-\frac{1}{Y_{-}}\right)\left(1-\frac{1}{Y_{+}}\right) \hat{\star} K^{y 1_{*}} .
\end{aligned}
$$

The kernels $K_{M 1_{*}}^{\Sigma}, K^{y 1_{*}}$ and $K_{v w x}^{M 1_{*}}$ with one leg on the real rapidity line of the mirror theory and the other one on the real line of string theory are given in [14]. The quantity $U$ appearing in the first line of the exact Bethe equation is defined as

$$
\mathrm{U}(v) \equiv 2 \int_{-\infty}^{+\infty} \mathrm{d} t \log \left[\prod_{j=1}^{N} S\left(u_{j}^{-}-t\right)\left(t-u_{j}\right)\right] K_{v w x}^{11 *}(t, v)-2 \sum_{j=1}^{N} \log \left(u_{j}-v-\frac{2 i}{g}\right) \frac{x_{j}^{-}-\frac{1}{x^{-}}}{x_{j}^{-}-\frac{1}{x^{+}}} .
$$

Finally, the kernel $\tilde{s}$ is defined as

$$
\tilde{s}(u, v) \equiv s\left(u-v-\frac{i}{g}+i 0\right) .
$$

The second variable of any kernel entering eq. (3.1) and the function $\mathrm{U}(v)$ should be evaluated at $v=u_{k}$. Only the imaginary part of the exact Bethe equations is written down in eq.(3.1), because using the explicit form of the kernels one can recognize that the real part vanishes, as it should be for the equation to have a real solution. Note that

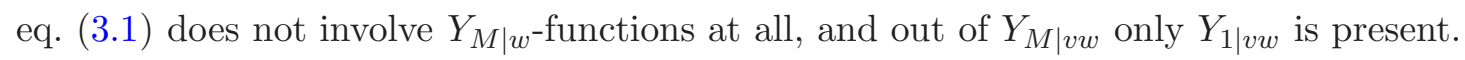

The exact Bethe equations can be written as

$$
\pi\left(2 n_{k}+1\right)=J p_{k}+i \sum_{j=1}^{N} \log S_{\mathfrak{s l}(2)}^{1_{*} 1_{*}}\left(u_{j}, u_{k}\right)+\mathcal{R}_{k} .
$$

Now we would like to apply the same strategy as the one in the TBA equations and find the leading weak coupling correction to the asymptotic value $\mathcal{R}_{k}^{\text {asympt }}=0$. The condition $\mathcal{R}_{k}^{\text {asympt }}=0$ must be satisfied for any $g$ to guarantee the compatibility of the exact Bethe equations with the asymptotic Bethe ansatz. Taking into account that for the Konishi state $J=2$ and $L=4$, we have checked numerically that this is indeed the case, for arbitrary values of $w \equiv u_{1}=-u_{2}$ inside the region where only $Y_{1 \mid v w}$ has two zeroes in the strip $|\operatorname{Im} v|<1 / g$. Thanks to this identity, we can remove the term $\delta w \partial_{w} U$ from the corrections to the asymptotic Bethe ansatz.

\footnotetext{
${ }^{2}$ According to $[38,39]$, the exact Bethe equations are $Y_{1 *}\left(u_{k}\right)=-1$, where $Y_{1 *}$ is the function $Y_{1}$ analytically continued to the real rapidity line of the string region. The exact Bethe equations which follow from the canonical TBA equation for $Y_{1}$ have been discussed in [12]. We exploit here the exact Bethe equations in a different representation which is derived from the hybrid TBA equation for $Y_{1}$ discussed in [14].
} 
Next, one can see that the leading weak-coupling correction, which we denote by $\mathcal{R}_{k}$ starts at $g^{8}$. It turns out that the last two lines in eq. (3.1) contain the convolution $\hat{\star}$ which, just as in the case of the TBA equations, will not contribute to the leading order of perturbation theory. Thus, at leading order we have the following shift of the asymptotic Bethe-Yang equations

$$
\delta \mathcal{R}_{k}=-2 \sum_{M=1}^{\infty} Y_{M}^{o} \star \operatorname{Im} K_{M 1_{*}}^{\Sigma}+2 A_{1 \mid v w} \mathscr{Y}_{1 \mid v w} \star \operatorname{Im} \tilde{s}+2 \sum_{M=1}^{\infty} Y_{M+1}^{o} \star s \star \operatorname{Im} K_{v w x}^{11_{*}} .
$$

Here all the kernels are evaluated at $g=0$ which provides the leading order contribution. In particular, we have

$$
\operatorname{Im} K_{v w x}^{11_{*}}=-\frac{1}{2 \pi}\left[\frac{u-v}{4+(u-v)^{2}}-\text { p.v. } \frac{1}{u-v}\right] .
$$

The convolution of the kernels in the last term of eq. (3.5) can be computed in terms of Digamma functions; for all $M \geq 1$ we find

$$
\begin{aligned}
s \star \operatorname{Im} K_{v w x}^{M 1_{*}}=\frac{1}{8 \pi i}[ & -i \frac{4(u-v)}{M^{2}+(u-v)^{2}} \\
& +\psi\left(\frac{M}{4}+\frac{i}{4}(u-v)\right)-\psi\left(\frac{M}{4}-\frac{i}{4}(u-v)\right) \\
& \left.-\psi\left(\frac{M+2}{4}+\frac{i}{4}(u-v)\right)+\psi\left(\frac{M+2}{4}-\frac{i}{4}(u-v)\right)\right] .
\end{aligned}
$$

Further, one needs $\operatorname{Im} \tilde{s}=$ p.v. $\frac{1}{4 \sinh \frac{\pi}{2}(u-v)}$. Finally, the leading contribution of the imaginary part of the dressing kernel in the mirror-string region is given by

$$
\operatorname{Im} K_{M 1_{*}}^{\Sigma}=-\frac{u-v}{2 \pi\left[(M+1)^{2}+(u-v)^{2}\right]} .
$$

The $\Phi$ and $\Psi$ functions which appear in the definition of the dressing phase kernel [40], do not contribute at this order, because they can be written as integrals over the interval $u \in[-2 g, 2 g]$ after the rescaling of rapidities. Combining now everything together, we read off the necessary correction ${ }^{3}$

$$
\begin{aligned}
\delta \mathcal{R}_{k}=\sum_{M=1}^{\infty} \int_{-\infty}^{\infty} \mathrm{d} u Y_{M}^{o}(u) \frac{1}{\pi} \frac{u-u_{k}}{(M+1)^{2}+\left(u-u_{k}\right)^{2}} \\
+\int_{-\infty}^{\infty} \mathrm{d} u A_{1 \mid v w}(u) \mathscr{Y}_{1 \mid v w}(u) \frac{1}{2 \sinh \frac{\pi}{2}\left(u-u_{k}\right)} \\
+\sum_{M=1}^{\infty} \int_{-\infty}^{\infty} \mathrm{d} u Y_{M+1}^{o}(u) \frac{1}{4 \pi i}\left[-i \frac{4\left(u-u_{k}\right)}{M^{2}+\left(u-u_{k}\right)^{2}}\right. \\
+\psi\left(\frac{M}{4}+\frac{i}{4}\left(u-u_{k}\right)\right)-\psi\left(\frac{M}{4}-\frac{i}{4}\left(u-u_{k}\right)\right) \\
\\
\left.-\psi\left(\frac{M+2}{4}+\frac{i}{4}\left(u-u_{k}\right)\right)+\psi\left(\frac{M+2}{4}-\frac{i}{4}\left(u-u_{k}\right)\right)\right]
\end{aligned}
$$

\footnotetext{
${ }^{3}$ In the second line of the equation below the function $1 / \sinh \left(u-u_{k}\right)$ exhibits singularity at $u=u_{k}$. We can however omit the principal value prescription for the integral because $A_{1 \mid v w}$ vanishes at $u=u_{k}$ making the integrand regular everywhere.
} 


\begin{tabular}{|c|l|l|l|l|}
\hline$M$ & TBA & Lüscher & TBA-Lüscher & $\frac{\text { TBA-Lüscher }}{\text { Lüscher }}$ \\
\hline 2 & -0.0108303 & -0.0108304 & $5.21829 \cdot 10^{-8}$ & $4.81819 \cdot 10^{-6}$ \\
3 & -0.000118621 & -0.000118665 & $4.34822 \cdot 10^{-8}$ & 0.000366429 \\
4 & $-4.63638 \cdot 10^{-6}$ & $-4.6417 \cdot 10^{-6}$ & $5.32116 \cdot 10^{-9}$ & 0.00114638 \\
5 & $-3.78671 \cdot 10^{-7}$ & $-3.79407 \cdot 10^{-7}$ & $7.35899 \cdot 10^{-10}$ & 0.0019396 \\
6 & $-4.89345 \cdot 10^{-8}$ & $-4.94077 \cdot 10^{-8}$ & $4.73166 \cdot 10^{-10}$ & 0.00957676 \\
\hline
\end{tabular}

Table 1. Comparison of the numerical results. The table describes a numerical comparison between $\delta \mathcal{R}_{1}$ and $-\Phi^{(8)}\left(u_{1}\right)$. In the column "TBA" the results for the contributions to $\delta \mathcal{R}_{1}$ produced by individual $Y_{M}$ 's are presented for $M=2, \ldots, 6$. Analogously, the column "Lüscher" represents the same quantities but for $-\Phi^{(8)}\left(u_{1}\right)$. The contributions of the first line in (3.9) and the first term in (3.11) are not included in the table. The other two columns give the absolute and relative estimates for the difference between the TBA and Lüscher approaches.

In [30] the leading correction to the asymptotic Bethe-Yang equations was denoted by $\Phi^{(8)}$. Comparison of our definitions with that of [30] shows that the agreement between the Lüscher and the TBA approaches relies on the fulfillment of the following equality

$$
\delta \mathcal{R}_{k}+\Phi^{(8)}\left(u_{k}\right)=0
$$

where $u_{1}=w=\frac{1}{\sqrt{3}}=-u_{2}$ is the one-loop rapidity for the two-particle state corresponding to the Konishi operator.

According to [30], the quantity $\Phi^{(8)}$ was found to be

$$
\begin{aligned}
\Phi^{(8)}\left(u_{k}\right)= & \sum_{M=1}^{\infty} \int_{-\infty}^{\infty} \mathrm{d} u Y_{M}^{o}(u) \times \\
& \times \frac{1}{\pi}\left[-\frac{u-u_{k}}{(M+1)^{2}+\left(u-u_{k}\right)^{2}}-\frac{u-u_{k}}{(M-1)^{2}+\left(u-u_{k}\right)^{2}}+\frac{u_{k}}{-1+M^{2}+u^{2}-u_{k}^{2}}\right] .
\end{aligned}
$$

Apparently, formulae eqs. (3.9) and (3.11) look rather different, only the first line in eq. (3.9), which is due to the contribution of the dressing kernel, cancels in the sum with the first term in eq. (3.11).

Plugging in eq. (3.9) the representation (2.16) specified for $\mathscr{Y}_{1 \mid v w}$, we see that the last two lines in (3.9) never involve $Y_{1}^{o}$. The last two terms in eq. (3.11) exhibit the same phenomenon: for $M=1$ they cancel each other. This suggests to compare individual terms in eqs. (3.9), (3.11) associated with a contribution of a given $Y_{M}$. The case of $M=1$ trivially matches, and the term with $\mathscr{Y}_{1 \mid v w}$ should be understood as $\mathscr{Y}_{1 \mid v w}^{[M]}$ defined in (2.17). Performing numerical evaluation of the corresponding quantities, we summarized the results in table 1 for $M=2, \ldots, 6$.

Albeit looking different, the individual $Y_{M}$-contributions show a good agreement between $\delta \mathcal{R}_{k}$ and $-\Phi^{(8)}$. Increasing $M$ the precision tends to decrease, but this is because the contributions corresponding to higher $M$ become rather small and their evaluation requires more time. 
We conclude this paper with an observation to be understood in future. On the one hand, the expression (3.11) can be concisely written as

$$
\Phi^{(8)}\left(u_{1}\right)=-\Phi^{(8)}\left(u_{2}\right)=-\sum_{M=1}^{\infty} \int_{-\infty}^{\infty} \frac{\mathrm{d} u}{4 \pi} \partial_{w} Y_{M}^{o}(u),
$$

by using (2.9). Here the level-matching condition is imposed before the differentiation. The right hand side involves the derivative of the transfer matrix, and looks similar to the known result for the $\mathrm{O}(4)$ model [41]. On the other hand, the generalized Lüscher formula proposed in [30] involves the trace of the derivative of the S-matrix with respect to the mirror rapidity $u$. The two quantities coincide for the present problem of Konishi at five loops, but must be distinguished in the general situation. ${ }^{4}$

\section{Acknowledgments}

We thank Zoltan Bajnok and Juan Maldacena for interesting discussions. The work of G. A. was supported in part by the RFBR grant 08-01-00798-a, by the grant NSh-672.2006.1, by NWO grant 047017015 and by the INTAS contract 03-51-6346. The work of S.F. was supported in part by the Science Foundation Ireland under Grants No. 07/RFP/PHYF104 and 09/RFP/PHY2142. The work of R.S. was supported by the Science Foundation Ireland under Grants No. 07/RFP/PHYF104.

Open Access. This article is distributed under the terms of the Creative Commons Attribution Noncommercial License which permits any noncommercial use, distribution, and reproduction in any medium, provided the original author(s) and source are credited.

\section{References}

[1] A.B. Zamolodchikov, Thermodynamic Bethe ansatz in relativistic models. Scaling three state Potts and Lee-Yang models, Nucl. Phys. B 342 (1990) 695 [SPIRES].

[2] J. Ambjørn, R.A. Janik and C. Kristjansen, Wrapping interactions and a new source of corrections to the spin-chain/string duality, Nucl. Phys. B 736 (2006) 288 [hep-th/0510171] [SPIRES].

[3] G. Arutyunov and S. Frolov, On String S-matrix, Bound States and TBA, JHEP 12 (2007) 024 [arXiv:0710.1568] [SPIRES].

[4] G. Arutyunov and S. Frolov, String hypothesis for the $A d S_{5} \times S^{5}$ mirror, JHEP 03 (2009) 152 [arXiv:0901.1417] [SPIRES].

[5] G. Arutyunov and S. Frolov, Thermodynamic Bethe Ansatz for the AdS $\times S^{5}$ Mirror Model, JHEP 05 (2009) 068 [arXiv:0903.0141] [SPIRES].

[6] G. Arutyunov and S. Frolov, Simplified TBA equations of the $A d S_{5} \times S^{5}$ mirror model, JHEP 11 (2009) 019 [arXiv:0907.2647] [SPIRES].

[7] D. Bombardelli, D. Fioravanti and R. Tateo, Thermodynamic Bethe Ansatz for planar AdS/CFT: a proposal, J. Phys. A 42 (2009) 375401 [arXiv:0902.3930] [SPIRES].

\footnotetext{
${ }^{4}$ R.S. thanks Zoltan Bajnok and Nikolay Gromov for a discussion on this point.
} 
[8] N. Gromov, V. Kazakov, A. Kozak and P. Vieira, Integrability for the Full Spectrum of Planar AdS/CFT II, [arXiv:0902.4458].

[9] N. Gromov, V. Kazakov and P. Vieira, Exact Spectrum of Anomalous Dimensions of Planar $\mathcal{N}=4$ Supersymmetric Yang-Mills Theory, Phys. Rev. Lett. 103 (2009) 131601 [arXiv:0901.3753] [SPIRES].

[10] A. Hegedus, Discrete Hirota dynamics for AdS/CFT, Nucl. Phys. B 825 (2010) 341 [arXiv:0906.2546] [SPIRES].

[11] S. Frolov and R. Suzuki, Temperature quantization from the TBA equations, Phys. Lett. B 679 (2009) 60 [arXiv: 0906. 0499] [SPIRES].

[12] N. Gromov, V. Kazakov and P. Vieira, Exact AdS/CFT spectrum: Konishi dimension at any coupling, arXiv:0906.4240 [SPIRES].

[13] N. Gromov, Y-system and Quasi-Classical Strings, JHEP 01 (2010) 112 [arXiv:0910.3608] [SPIRES].

[14] G. Arutyunov, S. Frolov and R. Suzuki, Exploring the mirror TBA, arXiv:0911.2224 [SPIRES].

[15] Z. Bajnok and R.A. Janik, Four-loop perturbative Konishi from strings and finite size effects for multiparticle states, Nucl. Phys. B 807 (2009) 625 [arXiv:0807.0399] [SPIRES].

[16] M. Lüscher, Volume Dependence of the Energy Spectrum in Massive Quantum Field Theories. 1. Stable Particle States, Commun. Math. Phys. 104 (1986) 177 [SPIRES].

[17] R.A. Janik and T. Lukowski, Wrapping interactions at strong coupling - the giant magnon, Phys. Rev. D 76 (2007) 126008 [arXiv:0708.2208] [SPIRES].

[18] Y. Hatsuda and R. Suzuki, Finite-Size Effects for Dyonic Giant Magnons, Nucl. Phys. B 800 (2008) 349 [arXiv:0801.0747] [SPIRES].

[19] J.A. Minahan and O. Ohlsson Sax, Finite size effects for giant magnons on physical strings, Nucl. Phys. B 801 (2008) 97 [arXiv: 0801.2064] [SPIRES].

[20] N. Gromov, S. Schäfer-Nameki and P. Vieira, Quantum Wrapped Giant Magnon, Phys. Rev. D 78 (2008) 026006 [arXiv:0801.3671] [SPIRES].

[21] J. Penedones and P. Vieira, Toy models for wrapping effects, JHEP 08 (2008) 020 [arXiv:0806.1047] [SPIRES].

[22] Y. Hatsuda and R. Suzuki, Finite-Size Effects for Multi-Magnon States, JHEP 09 (2008) 025 [arXiv:0807.0643] [SPIRES].

[23] Z. Bajnok, R.A. Janik and T. Lukowski, Four loop twist two, BFKL, wrapping and strings, Nucl. Phys. B 816 (2009) 376 [arXiv:0811.4448] [SPIRES].

[24] M. Beccaria, V. Forini, T. Lukowski and S. Zieme, Twist-three at five loops, Bethe Ansatz and wrapping, JHEP 03 (2009) 129 [arXiv: 0901.4864] [SPIRES].

[25] J. Gunnesson, Wrapping in maximally supersymmetric and marginally deformed $\mathcal{N}=4$ Yang-Mills, JHEP 04 (2009) 130 [arXiv:0902.1427] [SPIRES].

[26] M. Beccaria and G.F. De Angelis, On the wrapping correction to single magnon energy in twisted $\mathcal{N}=4$ SYM, Int. J. Mod. Phys. A 24 (2009) 5803 [arXiv:0903.0778] [SPIRES].

[27] F. Fiamberti, A. Santambrogio and C. Sieg, Five-loop anomalous dimension at critical wrapping order in $\mathcal{N}=4$ SYM, JHEP 03 (2010) 103 [arXiv:0908.0234] [SPIRES]. 
[28] F. Fiamberti, A. Santambrogio, C. Sieg and D. Zanon, Wrapping at four loops in $\mathcal{N}=4$ SYM, Phys. Lett. B 666 (2008) 100 [arXiv:0712.3522] [SPIRES].

[29] V.N. Velizhanin, The Four-Loop Konishi in $\mathcal{N}=4$ SYM, arXiv:0808.3832 [SPIRES].

[30] Z. Bajnok, A. Hegedus, R.A. Janik and T. Lukowski, Five loop Konishi from AdS/CFT, Nucl. Phys. B $\mathbf{8 2 7}$ (2010) 426 [arXiv: 0906.4062] [SPIRES].

[31] T. Lukowski, A. Rej and V.N. Velizhanin, Five-Loop Anomalous Dimension of Twist-Two Operators, Nucl. Phys. B 831 (2010) 105 [arXiv: 0912.1624] [SPIRES].

[32] L.N. Lipatov, High-energy asymptotics of multicolor QCD and exactly solvable lattice models, hep-th/9311037 [SPIRES].

[33] L.N. Lipatov, Asymptotic behavior of multicolor QCD at high energies in connection with exactly solvable spin models, JETP Lett. 59 (1994) 596 [Pisma Zh. Eksp. Teor. Fiz. 59 (1994) 571] [SPIRES].

[34] L.D. Faddeev and G.P. Korchemsky, High-energy QCD as a completely integrable model, Phys. Lett. B 342 (1995) 311 [hep-th/9404173] [SPIRES].

[35] A.V. Kotikov and L.N. Lipatov, DGLAP and BFKL evolution equations in the $\mathcal{N}=4$ supersymmetric gauge theory, Nucl. Phys. B 661 (2003) 19 [Erratum ibid. B 685 (2004) 19] [hep-ph/0208220] [SPIRES].

[36] N. Beisert, V. Dippel and M. Staudacher, A novel long range spin chain and planar $\mathcal{N}=4$ super Yang-Mills, JHEP 07 (2004) 075 [hep-th/0405001] [SPIRES].

[37] N. Beisert, The SU(2|2) dynamic S-matrix, Adv. Theor. Math. Phys. 12 (2008) 945 [hep-th/0511082] [SPIRES].

[38] P. Dorey and R. Tateo, Excited states by analytic continuation of TBA equations, Nucl. Phys. B 482 (1996) 639 [hep-th/9607167] [SPIRES].

[39] P. Dorey and R. Tateo, Excited states in some simple perturbed conformal field theories, Nucl. Phys. B 515 (1998) 575 [hep-th/9706140] [SPIRES].

[40] G. Arutyunov and S. Frolov, The Dressing Factor and Crossing Equations, J. Phys. A 42 (2009) 425401 [arXiv: 0904.4575] [SPIRES].

[41] N. Gromov, V. Kazakov and P. Vieira, Finite Volume Spectrum of $2 D$ Field Theories from Hirota Dynamics, JHEP 12 (2009) 060 [arXiv:0812.5091] [SPIRES]. 